\title{
Tm:fiber laser in-band pumping a cryogenically-cooled Ho:YAG laser
}

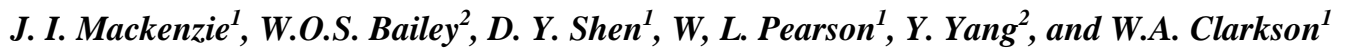 \\ ${ }^{1}$ Optoelectronics Research Centre, University of Southampton, Highfield, Southampton SO17 1BJ, U.K. \\ ${ }^{2}$ School of Engineering Sciences, University of Southampton, Highfield, Southampton SO17 1BJ, U.K.
}

jim@orc.soton.ac.uk

\begin{abstract}
:
Cryogenically-cooled diode-pumped lasers have received significant interest in recent years for their demonstrated orders of magnitude improvement in output radiance using simple laser resonator configurations, with respect to their room temperature counterparts. Here we present a technique that offers the potential for a further order of magnitude radiance improvement utilising the in-band pumping hybrid-laser architecture, which employs high-power fiber lasers to excite cryogenically-cooled bulk gain media. The ability to exploit the quasi-four-level nature of a two-level laser system at very cold temperatures enables the operation of very low quantum defect transitions, thus providing a tenfold reduction in the required thermal dissipation per unit power for the in-band pumped Ho:YAG laser discussed here, as compared to diode-pumped Yb:YAG. Preliminary results will be discussed, for a narrow linewidth Tm:fiber laser system operating in the 100W regime, pumping a cryogenically cooled Ho:YAG gain element, and employing a simple cavity configuration. Low quantum defect operation and power-scaling potential will be discussed.
\end{abstract}

\section{0 word abstract}

Cryogenically-cooled diode-pumped lasers have received significant interest in recent years for their demonstrated orders of magnitude improvement in output radiance using simple laser resonator configurations. Here we present a technique that offers the potential for a further order of magnitude improvement utilising the in-band-pumping hybrid-laser architecture to excite cryogenically-cooled bulk gain media. Preliminary results will be discussed, for a narrow linewidth Tm:fiber MOPA system operating in the 100W regime, pumping a cryogenically cooled Ho:YAG gain element, and employing a simple cavity configuration. Low quantum defect operation and power-scaling potential will be discussed. 